\title{
A penetração de luz nas águas de rios amazônicos
}

\author{
W. R. A. Muntz (*)
}

\section{Resumo}

Apresentam-se os resultados de mediçōes de penetração e de refletância de luz de 5 comprimentos de ondas nas águas de 7 localidades da Amazônia Central, perto de Manaus: água branca do rio Solimōes; água branca, decantada dum lago de várzea, lago Castanho, do Solimōes; água preta do rio Negro; água preta do rio Cuieiras, afluente do rio Negro. Água clara não se podia examinar. A menor penetração, mas com maior refletância, foi encontrada na água túrbida do Solimôes. No lago Castanho, a penetração aumentou com a distância do rio Solimōes, quer dizer, com o progresso da decantação. As maiores penetraçōes e menores refletâncias ocorreram nas águas pretas do rio Negro e, ainda mais, do rio Cuieiras, enquanto que a água a mais decantada do lago Castanho ocupava uma posição intermediária. Nas águas pretas, a penetração maior de luz de onda mais comprida era mais notável do que nas outras águas. O significado biológico dos resultados é discutido.

\section{INTRODUÇÃo}

As águas da Amazônia foram, há tempos, classificadas em três tipos básicos: águasbrancas, águas-pretas e águas-claras ou cristalinas (Wallace, 1889; Sioli, 1950). As águasbrancas, ou características dos rios Solimões e Amazonas, apresentam uma coloração forte devido aos depósitos de sedimentos aluvianos; há pouca penetração de luz nessas águas. Os rios de água preta contêm pouco ou nenhum limo, e neles a luz penetra melhor do que em águas-brancas, mas é colorida por material orgânico decomposto. O rio Negro é o maior exemplo desse tipo de águas. Finalmente, rios de águas-claras tais como o Tapajós contêm relativamente pouca quantidade de material or gânico decomposto ou em suspensão.

Uma série de análises químicas foram feitas com os diferentes tipos de água (e.g. Gessner, 1960; Sioli, 1950, 1965; Geisler et al., 1971; Santos et al., 1971), e os trabalhos também apresentam alguns dados sobre as condições de luz encontradas. Por exemplo, foi ob- servado que a medida do disco Secchi é tipicamente da ordem de $0,15 \mathrm{~m}$ em águas brancas, entre 1,0 e $1,5 \mathrm{~m}$ em águas-pretas, de $4 \mathrm{~m}$ ou mais em águas claras; em diversas localidades a cor da água foi medida em unidades de Platina. Foram feitas medidas de luz a direntes profundidades, em luz, em duas localidades do rio Madeira e do rio Negro, respectivamente (referências acima), mas não há medições detaihadas disponíveis. Exceto um único conjunto de medidas feito por Gessner (1960) no rio Negro, usando filtros de vidro de amplo espectro, não foram feitas medições sobre a penetração de luz de diferentes comprimentos de onda. O presente trabalho apresenta tais medidas feitas no rio Solimôes, em várias posições de um grande lago (Lago Castanho) ligado ao Solimões, no rio Negro, e em um afluente do rio Negro (Cuieiras). Não foi possível realizar mediçōes em águas claras.

\section{MÉTodos}

No campo foi usado o instrumento portátil descrito por Muntz \& Wainwright (1977). Foram feitas medições a cinco comprimentos de onda, selecionados por filtros de interferência, com uma média de $8.4 \mathrm{~mm}$ para a metade da amplitude máxima de faixa de onda. Um coletor de cosseno (e.g. Tyler \& Smith, 1970) foi ligado ao instrumento, e foram tomadas medidas de irradiação penetrante (com o instrumento virado para cima) e ressurgente (com o instrumento virado para baixo), tanto na superfície como em várais profundidades. Para a irradiação ressurgente uma profundidade de zero se refere a medidas feitas com o instrumento apontando para baixo, estando o coletor pouco abaixo da superfície. As medidas em laboratório foram feitas com o uso de um Espectrofotômetro Beckman DU.

As medidas no campo foram feitas em sete locais :

$\left({ }^{*}\right)$ - Department of Biology, University of Stirling, Stirling, FK9 4LA, Scotland. 
Local 1: fica no rio Solimões, à entrada de um canal em direção do lago Castanho, cerca de $50 \mathrm{~km}$ acima do encontro do rio Solimões com o rio Negro. As medidas foram tomadas em $17 / 01 / 77$.

Local 2 a 5: ficam em difeerntes posições do lago Castanho. O local 2 fica um pouco depois da entrada do lago e o local 5 fica no ponto mais distante possível de um dos braços do lago. Os locais 3 e 4 são intermediários. As medidas foram tomadas em 16/01/77.

Local 6: fica no rio Negro, cerca de 85 $\mathrm{km}$ acima de sua junção com o Solimões, um pouco acima da entrada do rio Cuieiras. As medidas foram tomadas em 27/01/77.

Local 7: fica no rio Cuieiras, aproximadamente $1 \mathrm{~km}$ acima de sua entrada no rio Negro. As medidas foram tomadas em $26 / 01 / 77$.

\section{RESULTADOS}

A Figura 1 mostra como a intensidade da iiuminação penetrante e ressurgente variou. de acordo com a profundidade, no local 5, o mais claro que se mediu no lago Castanho. Pode ser observado que o logarítmo da irradiação penetrante decresceu quase linearmente com a profundidade, conforme esperado de uma massa uniforme de água. Em nenhum dos 7 locais houve registros de qualquer descontinuidade com a profundidade; as relações entre - logarítmo da irradiação penetrante e a profundidade foram lineares em todas as medições.

A Figura 2 mostra, para cada local e para cada um dos cinco comprimentos de onda usados, a profundidade na qual a irradiação penetrante ficou reduzida a $1 \%$ de seu valor na superfície. A irradiação a uma dada profundidade (Iz) é obtida por meio da seguinte fórmula :

$$
\mathrm{l} z=\mathrm{I}_{\mathrm{o}} \mathrm{e}^{-\mathrm{kz}}
$$

onde $\mathrm{l}_{\mathrm{o}}$ é a iluminação na superfície, $z$ é a profundidade, e k é o coeficiente de atenuação difusa da îrradiação penetrante. Portanto, os valores de $\mathrm{k}$ podem ser imediatamente calculados a partir dos dados, e são representados

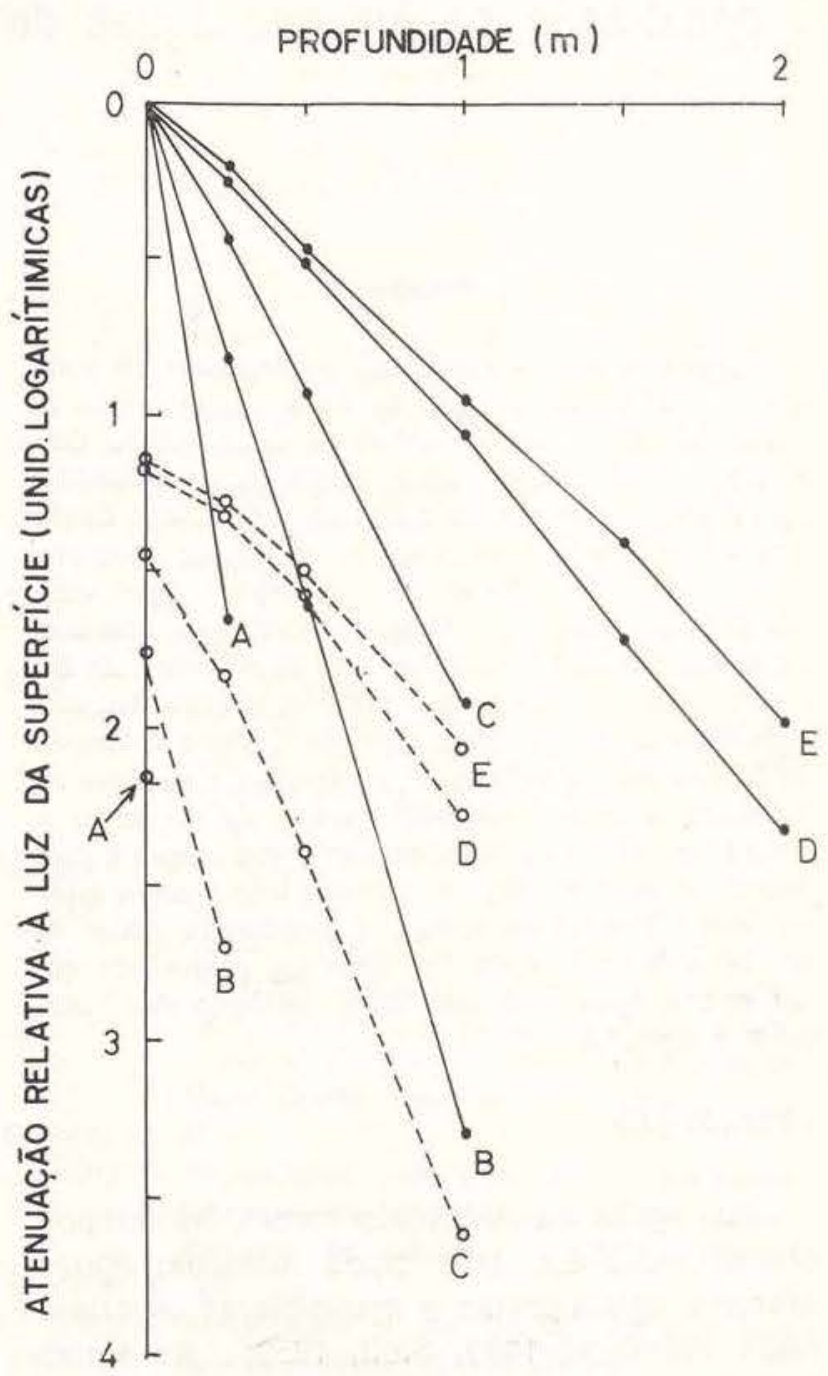

Fig. 1 - Atenuação da irradiação penetrante (linhas contínuas e círculos cheios) e ressurgente (linhas pontilhadas e círculos vazios) de acordo com a profundidade. Os resultados a $387.5 \mathrm{~nm}, 451.5 \mathrm{~nm}, 510 \mathrm{~nm}, 578 \mathrm{~nm}$, e $631.5 \mathrm{~nm}$ estão classificados de $A$ a $E$, respectivamente. A $387.5 \mathrm{~nm}$ a luz ressurgente só pode ser medida a zero metros: abaixo disso não houve luz suficiente para o instrumento registrar. Dados do lago Castanho, local 5 .

pelos eixos verticais do lado direito da figura. A iluminação a qualquer profundidade pode ser calculada com base nesses valores.

Quando as medições foram feitas o rio estava subindo e as águas estavam passando do rio Solimões para os lagos, inclusive o lago Castanho. O limo do rio Solimões deposita-se progressivamente nos lagos, de maneira que a água se torna mais clara conforme a dis- 


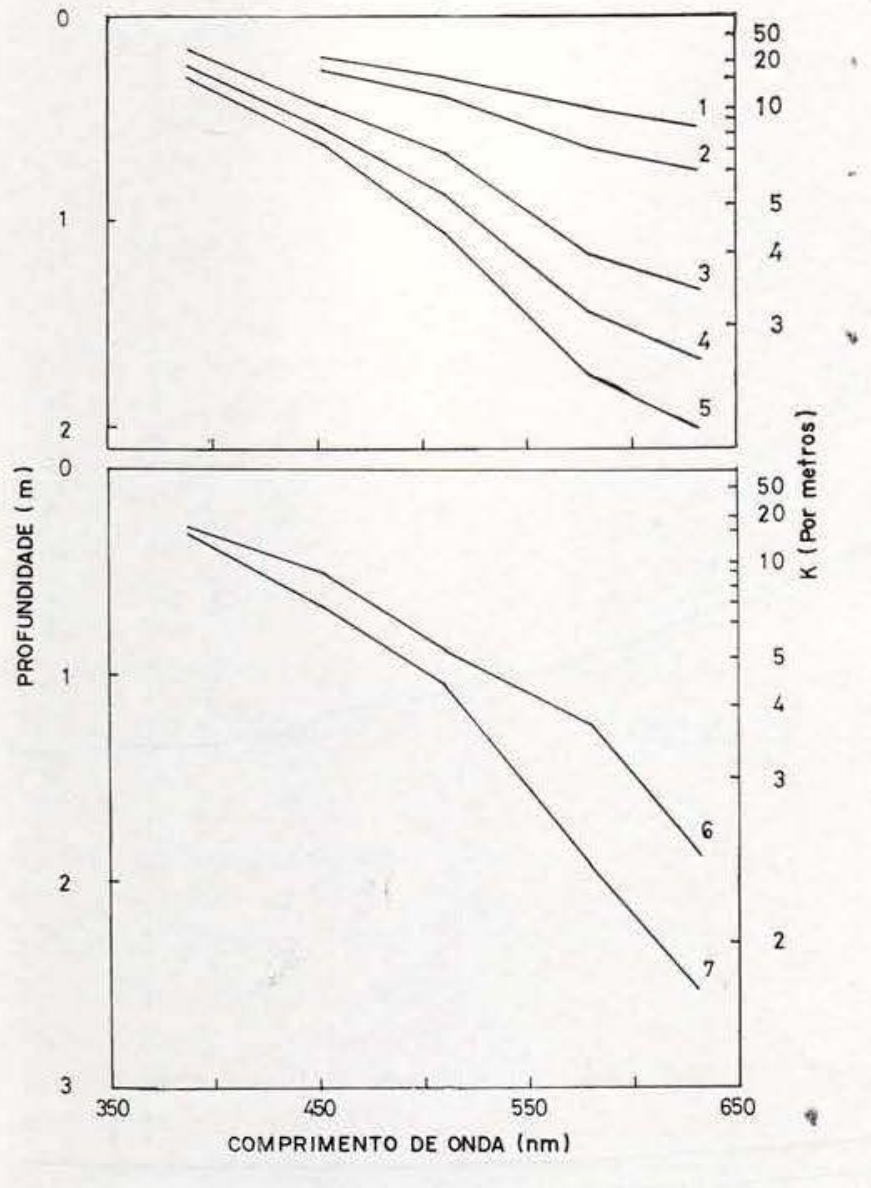

Fig. 2 - Irradiação penetrante a diferentes comprimentos de onda em diferentes locais. O eixo à esquerda mostra a profundidade, em metros, na qual a irradiação fica reduzida a $1 \%$ de seu valor na superfície, e o eixo à direita mostra os valores correspondentes do coeficiente de atenuação da penetração difusa, K. O local 1 fica no Solimões, os locais 2 a 5 no lago Castanho, o local 6 no rio Negro e o local 7 no rio Cuieiras. Consultar o texto para mais detalhes sobre os locais.

tância da entrada do lago aumenta. Isto poule ser claramente observado nas medições feitas nos locais 1 a 5 . A penetração de luz no rio Negro foi muito maior do que no rio Solimões, em todos os comprimentos de onda, e comparável com o local mais claro do lago Castanho. Entretanto, a forma da curva foi diferente, com uma penetração relativamente maior de luz de ondas longas. O rio Cuieiras foi mais claro do quue o rio Negro, embora a relação geral entre o comprimento de onda e a penetração seja semelhante nos dois rios.
A diferença entre o Solimôes (local 1) e o lago Castanho (local 5) foi devida ao material em suspensão, como pode ser observado na Figura 3. Isto mostra a absorbância de $1 \mathrm{~cm}$ de água dos vários locais, conforme medido em um Espectrofotômetro Beckman DU, tanto antes como depois de $20 \mathrm{~min}$. de centrifugação a 8.500 G. A centrifugação, ao remover o material em suspensão, reduziu a absorbância das amostras de água do rio Solimões e do lago Castanho (local 5), tornando-a idêntica em ambos os casos. Isto indica que a contribuição de material dissolvido foi igual em ambos locais e que a diferença se deve somente ao material em suspensão. A centrifugação da água do rio Negro não produziu efeito, indicando que o material em suspensão não contribuiu para a absorbância. Entretanto, parece que o rio Negro contém uma quantidade de material dissolvido maior do que a do Solimões ou do lago Castanho, desde que sua absorbância foi mais alta após a centrifugação.

Observe-se, porém, que os resultados obtidos em laboratório não são diretamente comparáveis às medidas tomadas no campo, devido às seguintes razões. No espectrofotômetro, a luz que se dispersa da amostra não alcança o fotodetector, e entå̃o não é medida. No instrumento usado no campo, a luz que alcançaria o instrumento, mas que se perde por causa da dispersão, é compensada pela luz extra proveniente de partes vizinhas da massa de água. Portanto, se as perdas de luz por absorção serão aproximadamente as mesmas em ambos os tipos de medidas, as perdas através de dispersão afetarão as medições em laboratório muito mais do que no campo. As medições no campo são mais úteis para a compreensão de problemas biológicos.

Weinberg (1976) discute as relações entre diferentes tipos de medidas de luz em água e também fornece referências a estudos anteriores.

Medições de irradiação ressurgente também foram feitas, e a Figura 1 mostra como essas medidas decrescem com a profundidade no local 5. A Figura 4 mostra a proporção entre a luz ressurgente e a luz penetrante (a reflectância), em unidades logarítmicas, para quatro locais. Em geral houve pouca luz res- 


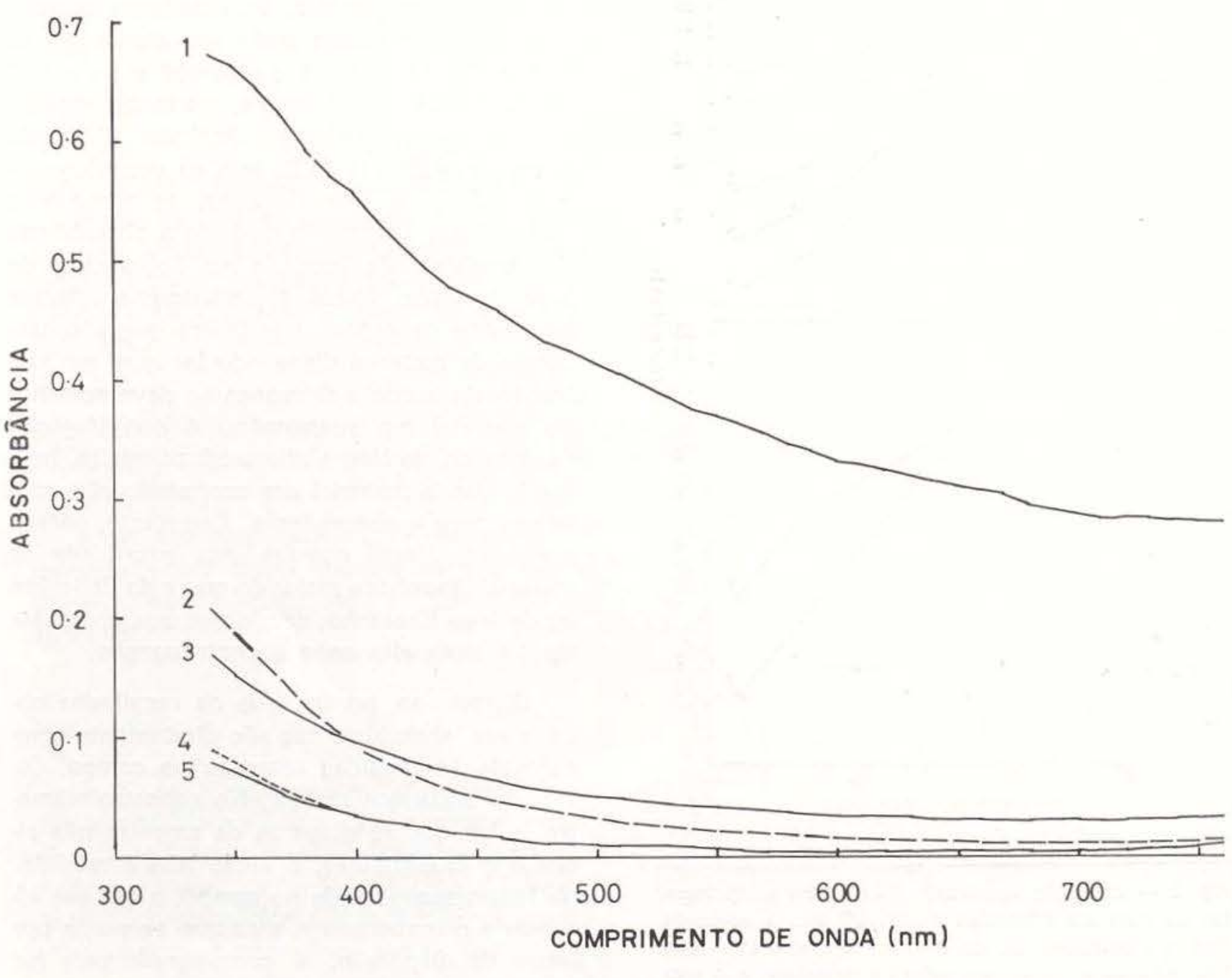

Fig. 3-Mediçōes da absorbância espectral, em laboratório, de diferentes amostras de água. A curva 1 representa a água do Solimões (loca| 1) antes da centrifugação; a curva 2 a água do rio Negro (local 6); a curva 4 do lago Castanho (local 5); a curva 5 do Solimões (local 1). As curvas 4 e 5 são indistinguíveis a comprimentos de onda maiores do que $400 \mathrm{~nm}$. Também foram feitas medições na água do rio Negro após centrifugação: os resultados não diferem dos representados pela curva 2 .

surgente, que só pode ser medida em águas rasas. A fiqura mostra as reflectâncias na superfície e nas profundidades para as quais foi possivel obter dados relativamente completos.

A reflectância foi consideravelmente maior quando a água continha material em suspensão (e.g. Solimões), provavelmente por causa do retro-espalhamento das partículas. A reflectância do"rio Negro foi muito baixa, $\alpha$ que colabora para sua aparência escura. Em todos os casos a reflectância foi maior em compri- mentos de ondas longas, isto é, a luz ressurgente conteve relativamente mais energia de ondas longas do que a luz penetrante.

\section{DISCUSSÃo}

As medições de luz foram feitas durante a fase $V$ do cruzeiro que o navio Alpha-Helix realizou recentemente na Amazônia. Embora os dados sejam relevantes para problemas tais como produtividade de plantas, é importante 


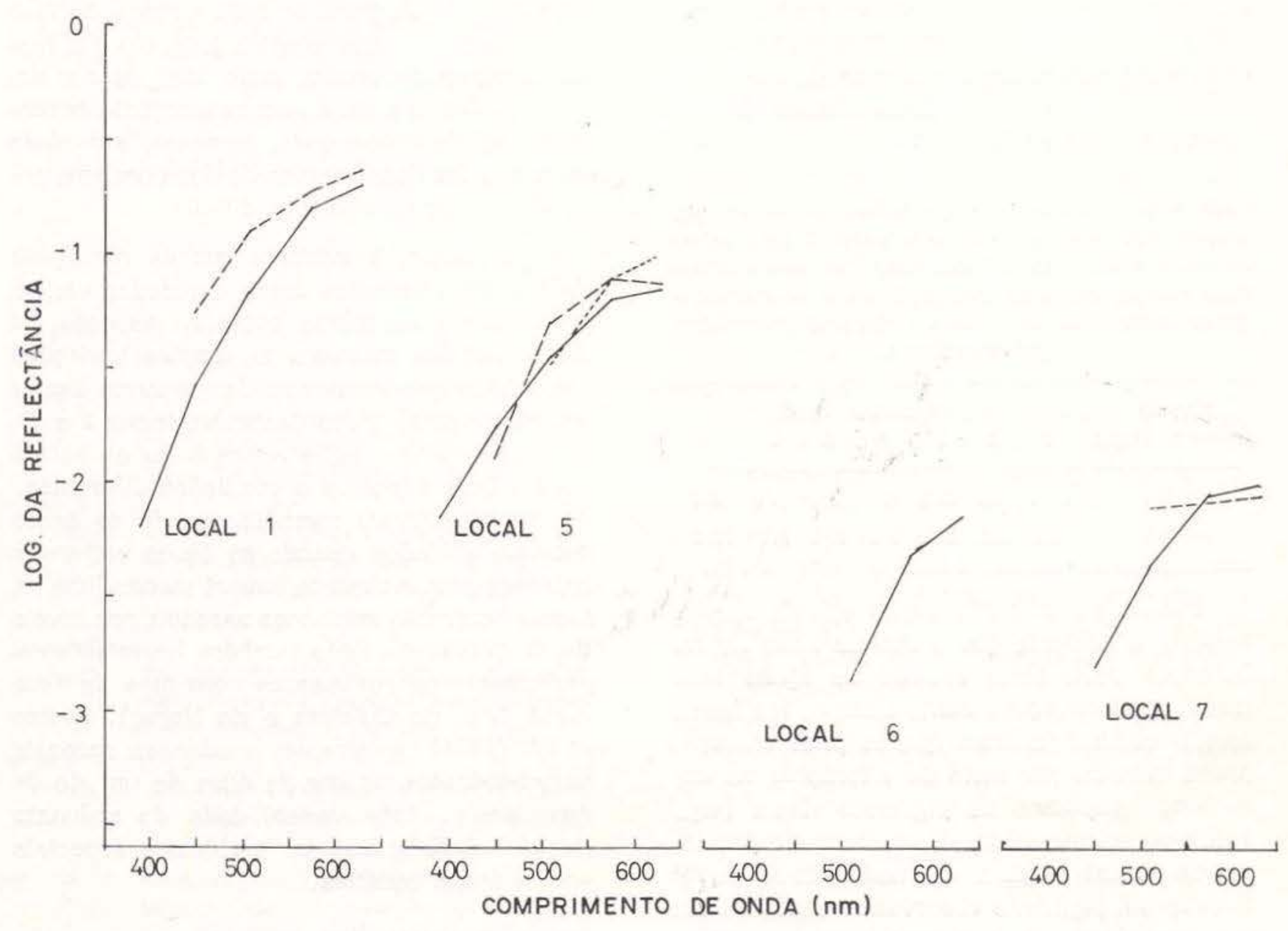

Fig. 4 - Curvas de reflectância para os vários locais. O eixo vertical representa o logaritmo da reflectância a uma dada profundidade (i.e. log. da irradiaçäo ressurgente - $\log$. da irradiação penetrante àquela profundidade). A linha continua representa a reflectância na superficie (zero m); a linha de traços longos representa a reflectância a $0,25 \mathrm{~m}$ e a linha de traços curtos a reflectância a $0,5 \mathrm{~m}$. Em muitos locais e profundidades a luz foi insuficiente para mediçōes em todos comprimentos de onda, e.g. o rio Negro (local 6), onde só foi possível medir nos três maiores comprimentos de onda à profundidade zero.

relacioná-los a problemas visuais, uma vez que a fase $\mathrm{V}$ foi amplamente voltada para o estudo dos mecanismos visuais dos peixes da região. Por exemplo, é possível fazer uma estimativa aproximada da maior profundidade na qual a visão ainda ocorre, como foi feita por Clarke (1936) em outras massas de água. Para isso é necessário conhecer a sensibilidade espectral dos peixes adaptados à escuridão, assim como seu limiar absoluto. Pelo menos entre as espécies desprovidas de cristalino amarelo e córneas, a sensibilidade espectral dependerá dos pigmentos visuais, cujos comprimentos de onda de absorbância máxima ( $\max$ ) variam entre 502nm e 535nm (Muntz, 1973). Não há dados disponiveis sobre os limiares absolutos de nenhuma espécie de peixe da Amazônia. Os dados sobre o comportamento de Lepomis ("sunfish") indicam um limiar de cerca de $10^{-6}$ mililamberts a comprimentos de onda entre 530 a $550 \mathrm{~nm}$ (Grunddfest, 1932), que é comparável ao limiar absoluto de seres humanos Os dados sobre o comportamento de dois ciprinídeos, obtidos por Muntz \& Northmore 1973) e Northmore (1977), indicam que esses peixes também apresentam limiares absolutos próximos ao do homem, para o qual a plena luz do dia é cerca de $10^{10}$ vezes o valor do limiar 
absoluto. Fazendo uma estimativa aproximada podemos considerar que a maior profundidade na qual um peixe da Amazônia pode ver é dez unidades logarítmicas menos do que a luz absorvida na superfície. Os resultados das es. timativas estão na Tabela 1.

TABELA 1 - Profundidades máximas estimadas, em metros, nas quais a visão seria possível para peixes em vários locais. Os cálculos foram feitos considerando-se que os pigmentos visuais absorvem ao máximo a $502 \mathrm{~nm}$ e $535 \mathrm{~nm}$. Consultar o texto para informações sobre os locais.

\begin{tabular}{lcccccccc}
\hline \multirow{2}{*}{$\begin{array}{c}\text { Max do } \\
\text { pigmento visual }\end{array}$} & $\mathbf{1}$ & $\mathbf{2}$ & $\mathbf{3}$ & $\mathbf{4}$ & $\mathbf{5}$ & $\mathbf{6}$ & $\mathbf{7}$ \\
\hline $502 \mathrm{~nm}$ & 2.1 & 3.0 & 5.5 & 6.8 & 8.2 & 7.1 & 9.8 \\
$535 \mathrm{~nm}$ & 2.3 & 3.2 & 5.8 & 7.2 & 8.6 & 7.7 & 10.8 \\
\hline
\end{tabular}

Embora os cálculos sejam apenas aproximaçōes, é evidente que o uso da visão no rio Solımöes deve estar restrito às águas bem rasas. As atenuações muito altas de luz nessa área também significam que se pode alcançar pouca melhora por meio de alterações na absorbância espectral do pigmento visual (e.g., obtém-se apenas $20 \mathrm{~cm}$ de meihora na profundidade visual máxima estimada obtida se si tivesse um pigmento absorvendo a $535 \mathrm{~nm}$ em vez de $502 \mathrm{~nm}$ ). Pela mesma razão, a profundidade visual máxima também é surpreendenitmente pouco afetada pela sensibilidade absoiuta do animal: por exemplo, a redução de uma unidade logarítmica, no limiar adotado, causa uma redução de apenas $0.2 \mathrm{~m}$ na profunaidade visual máxima. Em águas-claras, não somente a profundidade visual máxima é maior, mas o efeito de alterações nas caracteristicas espectrais dos pigmentos visuais também aumenta.

A profundidade visual máxima é apenas um aspecto da visão: o peixe também enfrenta outros problemas que são mais difíceis de avaliar. No rio Negro, por exemplo, há pouca luz ressurgente, e então espera-se que a visão ao longo de eixos dirigidos para baixo seja muito difícil e requeira uma sensibilidade muito alta. Entretanto, em muitos casos, objetos colocados abaixo dô animal serão detectados porque refletem a luz da superfície, pois, parecem mais brilhantes do que o fundo. Em tais casos, uma baixa iluminação ressurgente da própria água, fazendo o fundo parecer mais escuro, de fato aumentará o contraste entre o objeto e o fundo, tornando-se, assim, mais fácil de ser detetável. O alto nível de luz ressurgente encontrado no Solimões pode, portanto, ser outro fator que faz dessa massa de água um ambiente visual particularmente difícil.

Concluindo, é evidente que as condições de luz nas diferentes áreas estudadas variam muito, tanto no tempo como no espaço. Os dados obtidos mostram as amplas variações que podem ocorrer dentro de um certo lago a um certo tempo (lago Castanho, locais 2 a 5 ). e ao se moverem entre essas áreas os peixes podem ficar expostos a condições diferentes. As medidas foram tomadas quando as águas estavam subindo: quando as águas estiverem baixando provavelmente haverá menos limo no lago e ocorrerão mudanças sazonais nos níveis de fitoplâncton. Pode também haver diferenças marcantes em massas contíguas de água preta (e.g. rio Cuieiras e rio Negro). Santos et al. (1971) mostraram mudanças sazonais surpreendentes na cor da água de um rio de água preta. Esta variabilidade do ambiente também deve apresentar problemas especiais para a fauna aquática.

\section{AGRADECIMENTOS}

O presente trabalho foi realizado em janeiro de 1977, durante a fase $V$ da expedição $R / V$ do Alpha-Helix pela Amazônia, apoiada financeiramente pela National Science Foundation. Expressamos nossos agradecimentos ao Science Research Council, por cobrir as despesas das viagens de ida e volta ao Brasil, e ao Dr. N. A. Locket, pela colaboração nas medições.

\section{SUMMARY}

The paper presents the results of measurements of penetration and reflection of light of 5 different wavelenghts in the waters of 7 localities of Central Amazonia around Manaus: White water of Rio Solimões; decanted white water of a floodplain lagoon "Lago Castanho" of Rio Solimões; black water of Rio Negro; black water of Rio Cuieiras, affluente of Rio Negro. No clearwater river could be examined. Smallest penetration but greatest reflection occurred in turbid Solimões water. In Lago Castanho, penetration increased with distance from Solimões, i.e. with increase of 
decantation. Greatest penetrations and smallest reflections were found in the black waters of Rio Cuieiras and Rio Negro while the most decanted water of Lago Castanho was between these values. In the black waters, the greater penetration of light of greater wavelenght was more pronounced than in white and decanted white waters. The biological significances of the results are being discussed.

\section{BIBLIOGRAFIA CITADA}

Clarke, G. L.

1936 - On the depth at which fishes can see. Ecology, 17: 452-546.

Geisler, R.; Knôppel, H. A. \& Siolt, H.

1971 - Ökologie der Süsswasserfische Amazoniens Stand und Zukunftsaufgabem der Forschung. Naturwissenschaften, 58 : 303-311.

GESSNER, F.

1960 - Limnologisches Unterssunchungen am $\mathrm{Zu}$ sammenfluss des Rio Negro und des Amazonas (Solimões). Int. Rev. ges. Hydrobiol., $45: 55-79$.

GRUNDFEST, $\mathrm{H}$.

1932 - The sensibility of the sun-fish, Lepomis, to monochromatic radiation of low intensities. J. Gen. Physioll, $15: 807-832$.

MUNTZ, W. R. A.

1973 - Yellow filters and the absorption of light by the visual pigments of some Amazonian fishes. Vision Res., 13 : 2235-2254.

MUNTZ, W. R. A. \& NORTHMORE, D. P. M.

1973 - Scotopic spectral sensitivity in a teleeost fish (Scardinius erythrophthalmus) adapted to different daylengths. Vision Res., 13: 245-252.
MUNTZ, W. R. A. \& WaINWRIGTH, A. W.

1977 - Annual cycles in the light environments and visual mechanisms of fishes. In: Rhythmic activity of fish - Thorpe, J. E. ed. Symposium of the Fisheries Society of the British Isle (in press).

NORTHMORE, D. P. M.

1977 - Spacial summation and light adaptation in the goldfish visual system. Nature, 268 : 450-451.

Santos, U. de M.; Santos, A. \& Brinkman, W. L. F.

1971 - A Composição química do Rio Preto da Eva-Amąônio. Estudo preliminar. Ciência e Cultura, 23 : 643-646.

SIOLI, H.

1950 - Das Wasser in Amazonasgebiet, Forschung u. Fortschritte, Berlin, 26:274-280.

1965 - Bermekungen zur Typologie amazonischer Flüsse. Amazoniana, 1: 74-83.

TYLER, J. E. \& SMTTH, R. C. ,

1970 - Measorements of spectral irradiance underwater. New York, Gordon and Breach.

WALLACE, A. R.

1889 - Travels on the Amazon and Rio Negro. London, Melbourne.

WEINBERG, $\mathrm{S}$.

1976 - Submarine daylight and ecology. Marine Biol., 37 : 291-304.

(Aceito para publicação em 19/04/78) 